\title{
CRITERIA FOR SOLVABILITY OF LEFT INVARIANT OPERATORS ON NILPOTENT LIE GROUPS ${ }^{1}$
}

BY

\author{
LAWRENCE CORWIN
}

\begin{abstract}
We define a special nilpotent Lie group $N$ to be one which has a 1-dimensional center, dilations, square-integrable representations, and a maximal subordinate algebra common to almost all functionals on the Lie algebra $\mathfrak{R}$. Every nilpotent Lie group with dilations imbeds in such a special group so that the dilations extend. Let $L$ be a homogeneous left invariant differential operator on $N$. We give a representation-theoretic condition on $L$ which $L$ must satisfy if it has a tempered fundamental solution and which implies global solvability of $L$. (The sufficiency is a corollary of a more general theorem, valid on all nilpotent $N$.) For the Heisenberg group, the condition is equivalent to having a tempered fundamental solution.
\end{abstract}

1. Introduction. Let $N$ be a connected, simply connected nilpotent Lie group, with Lie algebra $\mathfrak{N}$. We consider $\mathscr{Q l}(\mathfrak{N})$, the universal enveloping algebra of $\mathfrak{N}$, to be the distributions on $N$ concentrated at the identity $e$. Define the transpose $L \mapsto L^{t}$ to be the antiautomorphism on $\mathscr{U}(\mathfrak{N})$ given by $X \mapsto-X$ on $\mathfrak{R}$. Then elements of $\mathscr{U}(\mathfrak{R})$ can be regarded as left invariant differential operators on $C^{\infty}(N)$, with $L \phi=\phi * L^{t}$, or as right invariant differential operators via the map $\phi \mapsto L^{t} * \phi$. We shall generally use $X \phi$ to denote the left invariant action of $X$; the main exception is in the proof of Lemma 3.1.

We shall be concerned with questions about the solvability of $L$. As is customary, we say that:

(a) $L$ is locally solvable if there is a neighborhood $U$ of $e$ such that for every $\phi \in \mathcal{C}_{c}^{\infty}(U)$ there is a function $u \in \mathcal{C}^{\infty}(U)$ with $L u=\phi$ on $U$;

(b) $L$ is semiglobally solvable if for every compact set $K$ in $N$ and every $\phi \in \bigodot_{c}^{\infty}(N)$, there is a function $u \in \bigodot_{c}^{\infty}(N)$ such that $L u=\phi$ on $K$;

(c) $L$ is globally solvable if for all $\phi \in \mathcal{C}^{\infty}(N)$, there is a function $\phi \in \bigodot^{\infty}(N)$ with $L u=\phi$;

(d) $L$ has a fundamental solution if there is a distribution $u$ on $N$ such that $L u=\delta=\delta_{e}$ (the point mass at $e$ );

(e) $L$ has a tempered fundamental solution if the distribution $u$ of (d) can be chosen to be tempered.

Clearly (c) $\Rightarrow(\mathrm{b}) \Rightarrow(\mathrm{a})$ and (e) $\Rightarrow(\mathrm{d}) \Rightarrow(\mathrm{b}) \Rightarrow(\mathrm{a})$. It is also known that (b) $\Rightarrow$ (c); see [5] or [12]. When $N$ is Abelian, (c) and (e) both hold. (The original proofs are in

Received by the editors February 15, 1982.

1980 Mathematics Subject Classification. Primary 22E27, 58G05; Secondary 35A05.

${ }^{1}$ Supported by NSF Contract \# MCS78-02715. 
[18] and [24]; see also [3], [1], and [2].) Not all elements $L$ of $\mathscr{Q}(\mathfrak{N})$ satisfy (a); indeed, the first example of an unsolvable differential operator (given in [22]) was an element of $\mathscr{U}(\mathfrak{N})$, where $N$ is the 3-dimensional Heisenberg group. Thus criteria for solvability are of some interest.

In this paper, we shall establish a sufficient condition for semiglobal solvability on $N$. The condition is representation-theoretic; it can be regarded as an extension of a theorem in [23] to the setting in [6]. We then show that for a certain restricted class of groups $N$ and a restricted set of operators $L$, the same condition is necessary for the existence of a tempered fundamental solution. In a few cases-most noticeably the Heisenberg groups - this condition can also be shown to imply the existence of a tempered fundamental solution.

We now describe the restrictions on $N$ and $L$. First of all, $N$ is assumed to be equipped with a 1-parameter family of dilations; that is, we can write $\mathfrak{N}=\bigoplus_{j=1}^{k} \mathfrak{N}_{j}$ (as a vector space) so that for all $a>0$, the map $A_{a}$ on $\mathfrak{N}$, defined by

$$
A_{a} \mid \mathfrak{N}_{j}=a^{j} \circ \text { identity, }
$$

is a Lie algebra automorphism. (Note that $\mathfrak{R}_{k}$ is in the center of $\mathfrak{R}$.) The maps $A_{a}$ extend to $\mathcal{U}(\mathfrak{N})$ and lift to $N$. Let $\mathscr{U}(\mathfrak{N})_{m}=\left\{D \in \mathcal{U}(\mathfrak{N}): A_{a}(D)=a^{m} D, \forall a>0\right\}$. An element of $\mathscr{U}(\mathfrak{N})$ is called homogeneous of degree $m$ if it is in $\mathscr{U}(\mathfrak{R})_{m}$. We can easily obtain a homogeneous basis for $\mathcal{U}(\mathfrak{R})$ by choosing one for $\mathfrak{R}$ and using the Poincaré-Birkhoff-Witt Theorem. Pick such a basis, $\mathcal{Q}$, and fix it for the rest of the discussion. Given an irreducible representation $\pi$ of $N$ on a Hilbert space $\mathcal{H}_{\pi}$, we let $\mathcal{H}_{\pi}^{\infty}$ be the space of $\mathcal{C}^{\infty}$ vectors and define the $s$ th Sobolev norm, \|\|$_{\pi, s}$, on $\mathcal{H}_{\pi}^{\infty}$ by

$$
\|v\|_{\pi, s}^{2}=\sum_{X \in \mathcal{Q}, \operatorname{degree}(X) \leqslant s}\|\pi(X) v\|^{2}, \quad s \geqslant 0 .
$$

$\mathcal{H}_{\pi}^{s}$ is the completion of $\mathcal{H}_{\pi}^{\infty}$ in the \|\|$_{\pi, s}$ norm, and $\mathcal{H}_{\pi}^{-s}$ is defined by duality. When $N$ does not have dilations, we can define Sobolev spaces similarly by using a filtration on $\mathfrak{N}$; see $\S 1.2$ of [16] for details. Ordinarily one realizes $\pi$ on $\mathcal{L}_{2}\left(\mathbf{R}^{n}\right)$ (for an appropriate $n$ ) by the Kirillov construction; according to Theorem 7.1 of [20], the Sobolev norms then determine the topology of $\delta\left(\mathbf{R}^{n}\right)$. (See also [7].)

Our main theorems apply only to certain nilpotent Lie groups with dilations. We say that $N$ is special if it has three additional properties:

(1) the center $Z$ of $N$ is one-dimensional;

(2) $N$ has square-integrable representations;

(3) there is an Abelian ideal $\mathfrak{M}$ of $\mathfrak{N}$ which is a polarizing subalgebra for "almost all" elements of $\mathfrak{R}^{*}$; moreover, $M$ has a homogeneous basis.

Some explanation of these propeties may help. Property (1) implies that $\operatorname{dim} N$ is odd; we let $\operatorname{dim} N=2 d+1$. Property (2) is discussed in [26]; it implies, in particular, that if $\pi$ is any irreducible representation of $N$ which is nontrivial on $Z$, then $\left.\pi\right|_{Z}$ determines $\pi$. It also implies that the Abelian ideal $\mathfrak{M}$ is $(d+1)$-dimensional.

Before stating the main theorems, we need one more piece of notation. The operator $\pi(L)$ maps $\mathcal{H}_{\pi}^{\infty}$ to $\mathcal{H}_{\pi}^{\infty}$; if $\mathcal{H}_{\pi}^{\infty}$ is imbedded in other spaces, $\pi(L)$ may be regarded as a (possibly unbounded) operator between these spaces. We write $\pi(L)$ : $\mathcal{H}_{\pi}^{s} \rightarrow \mathcal{H}_{\pi}^{t}$ for the closure of $\left.\pi(L)\right|_{\mathcal{F}_{\pi}^{\infty}}$. (In a slightly different context, Hörmander 
called this the "minimal operator".) On other occasions, we shall need to consider the operator which is the dual of $\pi\left(L^{t}\right): \mathcal{H}_{\pi}^{-t} \rightarrow \mathcal{H}_{\pi}^{-s}$; this operator (Hörmander's "maximal operator") is also an extension of $\left.\pi(L)\right|_{\mathcal{F}_{\pi}^{\infty}}$, and we shall write it as $\pi(L)_{m}$.

We now let $L$ be a homogeneous left invariant differential operator on the special Lie group $N$. When $\lambda \neq 0$, we write $\mathcal{H}_{\lambda}^{s}$ for $\mathcal{H}_{\pi_{\lambda}}^{s}$.

THEOREM 1.1. Suppose that there exists $s>0$ such that for all $\lambda \neq 0, \pi_{\lambda}(L)_{m}$, regarded as an operator from $\mathcal{H}_{\lambda}^{-s}$ to $\mathcal{H}_{\lambda}^{s}$, has a bounded right inverse. Then $L$ is semiglobally solvable.

THEOREM 1.2. Suppose that $L$ has a tempered fundamental solution. Then there exists $s>0$ such that for all $\lambda \neq 0, \pi_{\lambda}(L)_{m}: \mathcal{H}_{\lambda}^{-s} \rightarrow \mathcal{H}_{\lambda}^{s}$ has a bounded right inverse.

Theorems 1.1 and 1.2 fail to be converses exactly to the extent that global solvability differs from having a tempered fundamental solution (for these operators). It is reasonable to guess that operators $L$ satisfying the hypothesis of Theorem 1.1 actually have tempered fundamental solutions; in $\S 6$, we prove that this is the case if $N$ is the $(2 d+1)$-dimensional Heisenberg group. Thus we obtain a full converse to Theorem 1.2 in this case. We also prove a further (but rather weak) theorem along these lines.

We could also write Theorem 1.1 as follows: if there is an $s$ such that $\pi_{\lambda}\left(L^{t}\right)$ : $\mathcal{H}_{\lambda}^{-s} \rightarrow \mathcal{H}_{\lambda}^{s}$ has a bounded left inverse for all $\lambda \neq 0$, then $L$ is semiglobally solvable. Theorem 1.2 can be rephrased similarly. These restatements involve the minimal operator, and it is not true that if $L$ has a tempered fundamental solution, then $\pi_{\lambda}\left(L^{t}\right)_{m}: \mathcal{H}_{\lambda}^{-s} \rightarrow \mathcal{H}_{\lambda}^{s}$ is injective. An example is given in $\S 7$.

The $(2 n+1)$-dimensional Heisenberg groups are special, but other examples may not readily come to mind; indeed, special nilpotent Lie groups may seem rather too special to be interesting. We prove in $\$ 2$ that every nilpotent Lie group $N_{0}$ with dilations can be imbedded in a special nilpotent Lie group $N$ so that the dilations extend. (This result implies that there are square-integrable $n$-step nilpotent Lie groups for all $n$, as was claimed in [21].) If $L \in \mathcal{U}\left(\mathfrak{N}_{0}\right)$, then $L \in \mathcal{U}(\mathfrak{N})$, and $L$ is solvable on $N_{0}$ iff it is solvable on $N$. (See Theorem 52.6 of [35].) In some sense, therefore, Theorems 1.1 and 1.2 apply to all homogeneous left invariant operators $L$.

There have been a number of other papers concerned with solvability of left invariant operators. The situation for Abelian $N$ was described above. In [29], Raïs proved that for any nilpotent $N, L$ has a tempered fundamental solution whenever $L$ is bi-invariant; his proof was extended by Duflo in [11] to prove local solvability of bi-invariant operators on an arbitrary Lie group. An early example of a tempered fundamental solution for a homogeneous left invariant operator $L$ is found in [13]; this example was extended in [14]. See also [15]. $L$ is also hypoelliptic in these examples. In [30], Rockland conjectured Theorem 1.1 for the special case where $N$ is a Heisenberg group and $s=0$; the conjecture was proved in [31]. Extensions of this result were given in [6] and [33]; the latter gives a criterion like that of Theorem 1.1, 
but only for Heisenberg-type groups. The papers [21] and [32] give detailed information about local solvability of certain second order operators; earlier, the Lewy operator was analyzed in [17]. In [8], a necessary condition for local solvability is given; this condition is treated further in [33].

The organization of the rest of this paper is as follows: in $\$ 2$, we give the main properties of special nilpotent Lie algebras and prove that there are many examples. $\S 3$ is devoted to some auxiliary lemmas, after which, in $\$ 4$, we prove Theorem 1.2. Theorem 1.1 is proved in $\$ 5$; in fact, we prove a more general theorem that applies to arbitrary nilpotent Lie groups and restricts to Theorem 1.1 when $N$ is special. $\S 6$ contains results on fundamental solutions, and $\$ 7$ contains some examples and lists some unsolved problems.

We conclude this section by giving some notation. Whenever $\mathfrak{N}$ is a special nilpotent Lie algebra, we let $Y$ generate the center $z$ of $\mathfrak{N}$, and we assume that the degree of $Y$ is $m$. The homogeneous operator $L$ is usually taken to have degree $m_{0}$. We let $\operatorname{dim} N=2 d+1$.

We shall need a variety of Sobolev-like norms on $N$. Let $Q$ be as before; on $e_{c}^{\infty}(N)$, we define

$$
\|\phi\|_{p, s}^{p}=\sum_{X \in Q}{ }_{\operatorname{deg} X \leqslant s}\|\phi * X\|_{p}^{p}
$$

for $p=1,2$. The completion of $\bigodot_{c}^{\infty}(N)$ in the \|\|$_{p, s}$ norm will be written $\mathcal{C}_{p}^{s}(N)$. We also define

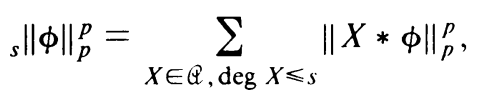

and let the completion be ${ }^{s} \complement_{p}(N)$. Finally, we let || be a homogeneous norm on $N$ (see [16] for an account), and let

$$
|\phi|_{s}^{2}=\sum_{X \in \mathbb{Q}, \operatorname{deg} X \leqslant s} \int_{N}(1+|x|)^{s}|\phi * X(x)|^{2} d x .
$$

The ||$_{s}$ norms are weighted Sobolev norms; $\mathcal{H}^{s}(N)$ is the completion of $\bigodot_{c}^{\infty}(N)$ in the ||$_{s}$ norm. (One could also use right invariant operators to define norms, but the left and right invariant weighted seminorms are equivalent for $\delta(N)$.) When $N$ does not have dilations, we replace the homogeneous norm with any standard norm on $N$.

I am greatly indebted to Professor F. P. Greenleaf for several very useful conversations.

2. Special nilpotent Lie groups. Suppose that $N$ is a special nilpotent Lie group of dimension $2 d+1$. We denote by $\mathfrak{N}$ its Lie algebra, by $\mathfrak{M}$ the Abelian ideal described in $\S 1$, and by $z$ the center of $\Re ; M, Z$ are the subgroups corresponding to $\mathfrak{M}, z$, respectively. Let $Y$ span $z$. We let $N_{2}$ be a Euclidean subspace in $N$, stable under the $A_{\lambda}$, such that every element of $N$ can be written uniquely as $x=x_{2} m$, $m \in M$, and $x_{2} \in N_{2}$, and we let $M_{1}$ be a subgroup of $M$ stable under the $A_{\lambda}$, $M_{1}=\mathbf{R}^{d}$, such that every element of $M$ can be written uniquely as $m=m_{1} z$, $m_{1} \in M_{1}$ and $z \in Z$. (If $X_{1}, \ldots, X_{2 d+1}$ is a homogeneous basis for $\mathfrak{R}$ such that $X_{1}=Y, \operatorname{span}\left\{X_{1}, \ldots, X_{j}\right\}$ is an ideal for each $j$, and $\operatorname{span}\left\{X_{1}, \ldots, X_{d+1}\right\}=\mathfrak{M}$, then 
one can take $M_{1}=\exp R X_{2} \cdots \exp R X_{d+1}, N_{2}=\exp R X_{d+2} \cdots \exp R X_{2 d+1}$ (See [25].) Set $N_{1}=M_{1} N_{2}$. Of course, $N_{1}$ and $N_{2}$ need not be subgroups.

As noted earlier, any irreducible representation of $N$ which is nontrivial on $Z$ is determined by its restriction to $Z$. We let $U_{\lambda}, \lambda \neq 0$, be the equivalence class or irreducible representations whose value on $\exp t Y$ is $e^{2 \pi i \lambda t} I$. We shall soon describe a particular element $\pi_{\lambda}$ of $U_{\lambda}$. The Kirillov orbit $\theta_{\lambda}$ corresponding to $U_{\lambda}$ consists of all elements $l \in \mathfrak{N}^{*}$ with $l(Y)=\lambda$. Define $\gamma(l)=l_{\mathfrak{m}}$, and fix some element $l_{\lambda} \in \Theta_{\lambda}$. Note that $\gamma\left(\Theta_{\lambda}\right)=\gamma\left(l_{\lambda}\right)+{ }^{\perp} \subset \mathfrak{M}^{*}$; that is, $\gamma\left(\Theta_{\lambda}\right)$ is a hyperplane in $\mathfrak{M}^{*}$.

Lemma 2.1. The map $x_{2} \mapsto\left(\operatorname{Ad}^{*} x_{2} \cdot l_{\lambda}\right)$ is a diffeomorphism of $N_{2}$ onto $\gamma\left(\theta_{\lambda}\right)$ and takes Lebesgue measure to a multiple of the canonical measure on $\theta_{\lambda}$ (see [28]).

Proof. If one regards $N_{2}$ as a cross-section of $N / M$, then Lebesgue measure on $N_{2}$ is the $N$-invariant measure for the obvious action of $N$. (See [36].) Similarly, $N$ acts on $\mathfrak{M}^{*}$ by $\mathrm{Ad}^{*}$, and Lebesgue measure on $\gamma\left(\theta_{\lambda}\right)$, the $\operatorname{Ad}^{*}(N)$-orbit of $\gamma\left(l_{\lambda}\right)$ is $N$-invariant; see [27, p. 54]. Next, if $x \in M$, then $\gamma\left(\operatorname{Ad}^{*} x \cdot l_{\lambda}\right)=\gamma(l)$. For if $W \in \mathfrak{M}$, then $\left(\left(\operatorname{Ad}^{*} x\right) l_{\lambda}\right) W=l_{\lambda}\left(\operatorname{Ad} x^{-1} \cdot W\right)=l_{\lambda}(W)$, because $M$ is Abelian and therefore Ad is trivial on $M$. Thus the map described above can be regarded as an $N$-covariant map from $N / M$ to $\gamma\left(\theta_{\lambda}\right)$, and we are done once we show that the stabilizer of $l_{\lambda}$ is $M$. This is a matter of counting. From [27, Theoreme, p. 50], the stabilizer of $l_{\lambda}$ is a connected subgroup $N(\lambda)$ of $N$ such that $\operatorname{dim} N / N(\lambda)=\operatorname{dim} \gamma\left(\theta_{\lambda}\right)=d=$ $\operatorname{dim}(N / M)$. As $M$ is connected, $N(\lambda)=M$.

We may fix $l_{\lambda}$ (at least on $M$ ) by assuming that $l_{\lambda}$ is trivial on $\mathfrak{M}_{1}=\log M_{1}$. Then the various $\gamma\left(l_{\lambda}\right)$ lie along a line. Fix Lebesgue measures $\mu$ on $N_{1}$ and $\nu$ on $z^{\perp} \subseteq \mathfrak{M}^{*}$; use this latter measure to define Lebesgue measures on the orbits $\gamma\left(\theta_{\lambda}\right)$. It is now easy to see that (up to a constant independent of $\lambda$ ) the map of Lemma 1 takes $\mu$ to $|\lambda|^{-d} \nu$. In particular, it is singular at $\lambda=0$. Elsewhere, it is a polynomial diffeomorphism with rational inverse.

Let $\alpha_{\lambda} f=C_{\lambda}\left(f \circ A_{\lambda_{0}}\right)$, where $\lambda_{0}=\lambda^{1 / m}$ and $C_{\lambda}$ is a constant to make $\alpha_{\lambda}$ unitary. We let $\sigma_{\lambda}^{\prime}$ be the representation induced from the representation on $Z$ taking $\exp t Y$ to $e^{2 \pi i \lambda t}$ (we regard $N_{1}$ as a cross-section for $Z \backslash N$ and let $\sigma_{\lambda}^{\prime}$ act on $\left(L_{2}\left(N_{1}\right)\right.$ ), and let $\sigma_{\lambda}=\alpha_{\lambda}^{-1} \sigma_{ \pm} \alpha_{\lambda}$, where $\sigma_{ \pm}=\sigma_{1}^{\prime}$ if $\lambda>0$ and $=\sigma_{-1}^{\prime}$ if $\lambda<0$. Then, of course, $\sigma_{\lambda}$ and $\sigma_{\lambda}^{\prime}$ are equivalent; indeed, $\alpha_{\lambda}$ is an intertwining operator.

We now choose an explicit representation $\pi_{\lambda}$ in each ${{ }_{\lambda}}_{\lambda}$. We do this by giving realization of $\pi_{1}$ and $\pi_{-1}$, and then letting $\pi_{\lambda}=\alpha_{\lambda}^{-1} \pi_{ \pm} \alpha_{\lambda}$. For $\pi_{1}$, define $\rho_{1}$ on $M$ by letting $\rho_{1}(\exp t Y)=e^{2 \pi i t}, \rho_{1} \mid M_{1}=1$. Now let $\pi_{1}=\operatorname{Ind}_{M \rightarrow N} \rho_{1}$, where we take $N_{2}$ as a cross-section for $M \backslash N$ and regard $\pi_{1}$ as acting on $N_{2}$. For $\pi_{-1}$, the construction is similar, but $\rho_{-1}(\exp t Y)=e^{-2 \pi i t}$. Note that if $L \in \mathscr{U}(\mathfrak{N})$ has degree $\mathfrak{M}_{0}$, then $\pi_{a \lambda}(L)=a^{m_{0} / m_{\lambda}} \pi_{\lambda}(L)$ for $a>0$.

LEMMA 2.2 The representation $\sigma_{\lambda}$ is equivalent to infinitely many copies of $\pi_{\lambda}$ (by a map which is described explicitly below).

Proof. The point of the lemma is that there is an explicit map decomposing $\sigma_{\lambda}$. We give the map for $\lambda=1$; a similar map works for $\lambda=-1$, and the result for arbitrary $\lambda$ follows from using the scaling maps $\alpha_{\lambda}$. 
Define $l_{1}$ as above, so that $l_{1}$ is trivial on $\mathfrak{M}_{1}$ and $l_{1}(Y)=1$; let $l_{y}=\operatorname{Ad}^{*}(y) l_{1}$, $y \in N_{2}$. Given $f \in \mathcal{L}_{2}\left(N_{1}\right)$, define $\beta_{1} f$ on $N_{2} \times z^{\perp}$ (here $z^{\perp}$ is a subspace of $\mathfrak{M}^{\perp}$ ) by

$$
\left(\beta_{1} f\right)\left(x, l_{y}-l_{1}\right)=\int_{M_{1}} f(m y x) e^{2 \pi i l_{y}(\log m+x(m))} d m,
$$

where $x(m) \in \mathfrak{M}$ is defined by $n^{-1} x^{-1} n x=\exp x(n)$. Then a calculation shows that

$$
\beta_{1} \sigma_{1}(x) \beta_{1}^{-1} f\left(x_{0}, l_{y}-l_{1}\right)=\pi_{1}(x) f\left(x_{0}, l_{y}-l_{1}\right),
$$

where $\pi_{1}$ acts on the first coordinate of $f$. That is,

$$
\beta_{1} \sigma_{1}(x) \beta_{1}^{-1}=\pi_{1} \otimes I \quad \text { on } \mathcal{L}_{2}\left(N_{2}\right) \otimes \mathcal{L}_{2}\left(z^{\perp}\right) \text {. }
$$

This gives the desired result.

LEMMA 2.3. Let $R$ be the right regular representation of $N$. Then $R$ is equivalent to a direct integral of the $\sigma_{\lambda}^{\prime}$ (by a map which is explicitly described below).

Proof. Define $\mathscr{F}: \mathfrak{L}_{2}(N) \rightarrow \mathcal{L}_{2}\left(N_{1} \times \mathbf{R}\right)$ as follows: if $x=x_{1} \exp t Y=\left(x_{1}, t\right)$, with $x_{1} \in N_{1}$, then

$$
(\mathscr{F} f)\left(x_{1}, \lambda\right)=\int_{\mathbf{R}} f\left(x_{1}, t\right) e^{-2 \pi i \lambda t} d t .
$$

Then $\mathscr{F}$ is unitary, and a calculation gives

$$
\left(\mathscr{F} R(x) \mathscr{F}^{-1}\right) f\left(x_{1}, \lambda\right)=\left(\sigma_{\lambda}^{\prime}(x) f\right)\left(x_{1}, \lambda\right),
$$

where $\sigma_{\lambda}^{\prime}$ acts on the first variable.

COROllaRy. There is a unitary map $\beta$ : $\mathcal{L}_{2}(N) \rightarrow \mathcal{L}_{2}\left(N_{2} \times z^{\perp} \times(\mathbf{R} \backslash\{0\})\right)$ which gives an equivalence between $R$ and the representation $\tau$ defined by

$$
\tau(x) f\left(n_{2}, l, \lambda\right)=\pi_{\lambda}(x) f\left(n_{2}, l, \lambda\right), \quad n_{2} \in N_{2}, l \in z^{\perp}, \lambda \in \mathbf{R} \backslash\{0\},
$$

where $\pi_{\lambda}$ acts on the first variable. Moreover, $\beta$ has the following property: if $\mathcal{T}=\left\{f_{2} \in \mathcal{S}(\mathbf{R}): \operatorname{supp} f_{2} \subseteq\left[\frac{1}{2}, 2\right]\right\}$, then

$$
\beta\left(\delta\left(N_{1}\right) \hat{\otimes} \mathcal{T}\right)=\delta\left(N_{2}\right) \hat{\otimes} \delta\left(z^{\perp}\right) \hat{\otimes} \mathcal{T}^{\wedge} .
$$

In fact, $\beta=\gamma \circ \mathcal{F}$, where $(\gamma \phi)(\cdot, \lambda)=\beta_{\lambda} \alpha_{\lambda} \phi(\cdot, \lambda)$. This last fact is easy to check because $\beta$ is given so explicitly.

Notice in particular that any function of the form $f_{0}\left(x_{2}\right) f_{1}\left(l_{y}-l_{1}\right) f_{2}(\lambda)$, where $f_{0}$, $f_{1}$, and $f_{2}$ are Schwartz class functions and supp $f_{2} \in\left[\frac{1}{2}, 2\right]$, can be written as $\beta f$ for some $f \in \mathcal{S}(N)$. In $\S 5$, we shall write $f=\left(f_{0}, f_{1}, f_{2}\right)$. If $L \in \mathcal{Q}(\mathfrak{N})$ is homogeneous of degree $m_{0}$, then (with $f_{0}, f_{1}, f_{2}$ as above)

$$
\left(\beta R(L) \beta^{-1}\right) f_{0} \otimes f_{1} \otimes f_{2}(x, l, \lambda)=\lambda^{m_{0} / m}\left(\pi_{1}(L)\right)\left(f_{0} \otimes f_{1} \otimes f_{2}\right)(x, l, \lambda) .
$$

We shall need to know the Plancherel formula for special nilpotent Lie groups $N$. We follow the treatment in [6]. Let $Y, X_{1}, \ldots, X_{d}$ be a basis for $\mathfrak{M}$, and let $X_{m+1}, \ldots, X_{2 d}$ extend this to a basis of $\mathfrak{R}$. Define $P f(l)$ (up to sign) by

$$
(P f(l))^{2}=\operatorname{Det}\left(l\left(\left[X_{i}, X_{j}\right]\right)\right) .
$$


Then $P f(l)$ depends only on the $\operatorname{Ad}^{*}(N)$-orbit of $l$, and hence only on $l(Y)$. Since we clearly have $(P f(a l))^{2}=a^{2 d} P f(l)$, we see that, up to a nonzero scalar,

$$
|P f(l)|=|l(Y)|^{d}, \quad 2 d+1=\operatorname{dim} N .
$$

We can therefore write the Fourier inversion formula $N$ : if $\phi \in S(N)$, then

$$
\operatorname{Tr} \pi_{\lambda}(\phi)=\int_{Y^{\perp}}|\lambda|^{-d} \phi\left(\lambda l_{0}+l^{\prime}\right) d l^{\prime}, \quad \phi(1)=\int_{\mathbf{R}-\{0\}}|\lambda|^{d} \operatorname{Tr} \pi_{\lambda}(\phi) d \lambda,
$$

where 1 is the identity of $N, d l^{\prime}$ is Lebesgue measure on $Y^{\perp} \subset \mathfrak{N}^{*}$, and

$$
\check{\phi}\left(l^{\prime}\right)=\int_{N} e^{2 \pi i l^{\prime}(X)} \phi(\exp X) d X
$$

Similarly,

$$
\|\phi\|_{2}^{2}=\int_{N}|\lambda|^{d} \operatorname{Tr}\left(\pi_{\lambda}(\phi)\left(\pi_{\lambda}(\phi)\right)\right) d \lambda .
$$

(This account can be generalized; see [6] and §5.)

Next, we prove that every Lie algebra with dilations can be imbedded in a special Lie algebra so that the dilations extend. We begin with a preliminary result.

Let $V=\bigoplus_{j=0}^{k} V_{j}$ be a graded vector space with $\operatorname{dim} V_{0}=\operatorname{dim} V_{k}=1$. For convenience, let $V_{j}=\{0\}$ if $j>k$. We let $\mathfrak{N}=\mathfrak{N}_{V}$ be the Lie algebra of all linear transformations $A: V \rightarrow V$ such that $v \in V_{i} \Rightarrow A v \in \bigoplus_{j=i+1}^{k} V_{j}$. (In particular, $A=0$ on $V_{k}$.) Let $\mathfrak{R}_{j}=\left\{A \in \mathfrak{R}: A\left(V_{i}\right) \subseteq V_{i+j}, 0 \leqslant i \leqslant k\right\}$. Then $\mathfrak{R}=\bigoplus_{j=1}^{k} \mathfrak{N}_{j}$ and $\left[\mathfrak{N}_{j_{1}}, \mathfrak{N}_{j_{2}}\right] \subseteq \mathfrak{N}_{j_{1}+j_{2}}$; hence $\mathfrak{N}$ is a nilpotent Lie algebra with dilations.

THEOREM 2.1. The algebra $\mathfrak{N}=\mathfrak{N}_{V}$ can be imbedded in a special nilpotent Lie algebra $\tilde{\mathfrak{N}}$ so that the dilations extend.

Proof. Let $e_{i, 1}, \ldots, e_{i}$, form a basis for $V_{i}$, and define operators $E\left(i_{1}, j_{1} ; i_{2}, j_{2}\right)$ : $V \rightarrow V$, for $i_{1}<i_{2}, j_{1} \leqslant n_{i_{1}}, j_{2} \leqslant n_{i_{2}}$, by

$$
\left[E\left(i_{1}, j_{1} ; i_{2}, j_{2}\right)\left(e_{i_{3}, j_{3}}\right)\right]=\delta_{i_{1}, i_{3}} \delta_{j_{1}, j_{3}} e_{i_{2}, j_{2}} .
$$

The elements $E\left(i_{1}, j_{1} ; i_{2}, j_{2}\right)$ form a basis for $\mathfrak{R}$, with

$$
\begin{aligned}
{\left[E\left(i_{1}, j_{1} ; i_{2}, j_{2}\right),\right.} & \left.E\left(i_{3}, j_{3} ; i_{4}, j_{4}\right)\right] \\
& =\delta_{i_{2}, i_{3}} \delta_{j_{2}, j_{3}} E\left(i_{1}, j_{1} ; i_{4}, j_{4}\right)-\delta_{i_{1}, i_{4}} \delta_{j_{1}, j_{4}} E\left(i_{3}, j_{3} ; i_{2}, j_{2}\right) .
\end{aligned}
$$

We let $\tilde{\mathfrak{R}}$ be spanned by the $E\left(i_{1}, j_{3} ; i_{2}, j_{2}\right)$ as above, plus elements $F\left(i_{1}, j_{1} ; i_{2}, j_{2}\right)$, with $1<i_{1}<i_{2} \leqslant k-1, j_{1} \leqslant n_{i_{1}}, j_{2} \leqslant n_{i_{2}}$, with brackets

$$
\begin{gathered}
{\left[E\left(i_{1}, j_{1} ; i_{2}, j_{2}\right), F\left(i_{1}, j_{1} ; i_{2}, j_{2}\right)\right]=E(0,1 ; k, 1) ;} \\
{\left[E\left(i_{2}, j_{2} ; i_{3}, j_{3}\right), F\left(i_{1}, j_{1} ; i_{4}, j_{4}\right)\right]= \begin{cases}\delta_{i_{3}, i_{4}} \delta_{j_{3}, j_{4}} F\left(i_{1}, j_{1} ; i_{2}, j_{2}\right) & \text { if } i_{1}<i_{2}, \\
0 & \text { if } i_{1} \geqslant i_{2} ;\end{cases} }
\end{gathered}
$$

all brackets of two $F$ 's are 0 . We let $\mathfrak{R}_{i}^{\tilde{i}}$ be spanned by the $E\left(i_{1}, j_{1} ; i_{2}, j_{2}\right)$ with $i_{2}-i_{1}=i$ and the $F\left(i_{1}, j_{1} ; i_{2}, j_{2}\right)$ with $i_{2}-i_{1}=k-i$.

We need first to verify that $\mathfrak{N}^{\tilde{i}}$ is a Lie algebra; for this, we need to verify the Jacobi identity on basis elements. For three $E$ 's, the Jacobi identity holds because it 
holds in $\mathfrak{R}$; for two or three $F$ 's, it holds because all three terms are 0 . Thus we need only verify that

$$
\begin{aligned}
0= & {\left[\left[E\left(i_{1}, j_{1} ; i_{2}, j_{2}\right), E\left(i_{3}, j_{3} ; i_{4}, j_{4}\right)\right], F\left(i_{5}, j_{5} ; i_{6}, j_{6}\right)\right] } \\
& +\left[\left[E\left(i_{3}, j_{3} ; i_{4}, j_{4}\right), F\left(i_{5}, j_{5} ; i_{6}, j_{6}\right)\right], E\left(i_{1}, j_{1} ; i_{2}, j_{2}\right)\right] \\
& +\left[\left[E\left(i_{5}, j_{5} ; i_{6}, j_{6}\right), E\left(i_{1}, j_{1} ; i_{2}, j_{2}\right)\right], E\left(i_{3}, j_{3} ; i_{4}, j_{4}\right)\right] .
\end{aligned}
$$

Assume first that $i_{2} \leqslant i_{3}$. We call the three brackets Terms 1,2, and 3, respectively, and try to evaluate them.

Term 3. If the inner bracket is nonzero and noncentral, then $i_{2}=i_{6}, j_{2}=j_{6}$, and $i_{5}<i_{1}$. In this case, Term 3 becomes $\left[F\left(i_{5}, j_{5} ; i_{1}, j_{1}\right), E\left(i_{3}, j_{3} ; i_{4}, j_{4}\right)\right]$. This bracket is zero unless $i_{1}=i_{4}$ and $i_{5}<i_{3}$. But since $i_{1}<i_{2}$ and $i_{3}<i_{4}$, we then have $i_{3}<i_{4}=i_{1}<i_{2}$, which contradicts the assumption. Hence Term 3 is 0 .

Term 1. We have $i_{1} \leqslant i_{2} \leqslant i_{3} \leqslant i_{4}$. Thus the inner bracket is 0 unless $i_{2}=i_{3}$ and $j_{2}=j_{3}$. In that case, Term 1 becomes $\left[E\left(i_{1}, j_{1} ; i_{4}, j_{4}\right), F\left(i_{5}, j_{5} ; i_{6}, j_{6}\right)\right]$. This bracket is 0 unless $i_{4}=i_{6}, j_{4}=j_{6}$, and $i_{5}<i_{1}$; in that case, it is $F\left(i_{5}, j_{5} ; i_{1}, j_{1}\right)$. Note that $i_{5}<i_{1}<i_{2}=i_{3}$.

Term 2. The inner bracket is 0 unless $i_{4}=i_{6}, j_{4}=j_{6}$, and $i_{5}<i_{3}$; then Term 2 becomes $\left[F\left(i_{5}, j_{5} ; i_{3}, j_{3}\right), E\left(i_{1}, j_{1} ; i_{2}, j_{2}\right)\right]$. This bracket is 0 unless $i_{3}=i_{2}, j_{3}=j_{2}$, and $i_{5}<i_{1}$; in that case, it is $-F\left(i_{5}, j_{5} ; i_{1}, j_{1}\right)$.

Thus Term 1 is nonzero exactly when Term 2 is nonzero, and in this case these terms cancel. Hence the Jacobi identity holds when $i_{2} \leqslant i_{3}$.

If $i_{4} \leqslant i_{1}$, a similar analysis applies; more simply, we can interchange $E\left(i_{1}, j_{1} ; i_{2}, j_{2}\right)$ and $E\left(i_{3}, j_{3} ; i_{4}, j_{4}\right)$ to see that the Jacobi identity holds. Finally, if $i_{2}>i_{3}$ and $i_{4}>i_{1}$, then the inner bracket in Term 1 is 0 ; also, the above analysis shows that Terms 2 and 3 are also 0 . It follows that $\mathfrak{N}^{\sim}$ is a Lie algebra.

It is easy to check that $\left[\mathfrak{N}_{i_{1}}^{\tilde{1}}, \tilde{\mathfrak{R}_{i_{2}}}\right] \subseteq \mathfrak{N}_{i_{1}+i_{2}}$. Thus $\tilde{\mathfrak{N}}$ has dilations. To find the center of $\mathfrak{\Re}$, we look at $[X, Y]$, where

$$
X=\sum a\left(i_{1}, j_{1} ; i_{2}, j_{2}\right) E\left(i_{1}, j_{1} ; i_{2}, j_{2}\right)+\sum b\left(i_{1}, j_{1} ; i_{2}, j_{2}\right) F\left(i_{1}, i_{2} ; j_{1}, j_{2}\right) .
$$

Suppose that one of the $a$ 's, other than $a(0,1 ; k, 1)$, is nonzero. If so, choose $i_{1}, i_{2}$ so that $a\left(i_{1}, j_{1} ; i_{2}, j_{2}\right) \neq 0, i_{1}$ is as large as possible given this condition, and $i_{2}$ is as small as possible given these conditions. If $i_{1}=0$, then

$$
\left[X, E\left(j_{1}, j_{2} ; k, 1\right)\right]=a\left(i_{1}, j_{1} ; i_{2}, j_{2}\right) E(0,1 ; k, 1) ;
$$

if $i_{2}=k$, then

$$
\left[X, E\left(0,1 ; i_{1}, j_{1}\right)\right]=-a\left(i_{1}, j_{1} ; i_{2}, j_{2}\right) E(0,1 ; k, 1) ;
$$

if $1<i_{1}<i_{2}<k$, then

$$
\left[X, F\left(i_{1}, j_{1} ; i_{2}, j_{2}\right)\right]=a\left(i_{1}, j_{1}, i_{2}, j_{2}\right) E(0,1 ; k, 1) .
$$

If all these $a$ 's are 0 , but some $b$ is nonzero, then choose $i_{1}, i_{2}$ so that $b\left(i_{1}, j_{1} ; i_{2}, j_{2}\right)$ $\neq 0, i_{2}$ is as large as possible given this condition, and $i_{1}$ is as small as possible given these conditions. Then

$$
\left[X, E\left(i_{1}, j_{1} ; i_{2}, j_{2}\right)\right]=-b\left(i_{1}, j_{1} ; i_{2}, j_{2}\right) E(0,1 ; k, 1) .
$$

Hence $E(0,1 ; k, 1)$ spans the center of $\mathfrak{N}$. 
The above calculation also shows that if $l \in(\mathfrak{N})^{*}$ is a linear functional that is nonzero on $E(0,1 ; k, 1)$, and if $X \in \mathbf{R}_{l}=\left\{X \in \mathfrak{R}^{\sim}: l([X, Y])=0, \forall Y \in \mathfrak{R}\right\}$, then $X$ is a multiple of $E(0,1 ; k, 1)$. Theorem 1 of [26] implies that $N$ has square-integrable representations. Since $\mathfrak{N}_{i} \subseteq \mathfrak{R}_{i}^{\tilde{}}$ for all $i$, the dilations extend. Finally, the span of the $F\left(i_{1}, j_{1} ; i_{2}, j_{2}\right)$ and the $E\left(i_{1}, j_{1} ; k, 1\right)$ is the required polarizing ideal. This finishes the proof.

Theorem 2.1 leads easily to the desired result.

COROLlaRY 2.2. Let $\mathfrak{R}=\bigoplus_{j=0}^{k} \mathfrak{N}_{j}$ be a nilpotent Lie algebra with dilations. Then $\mathfrak{N}$ can be imbedded in a special Lie algebra $\tilde{\mathfrak{N}}$ so that the dilations extend. Moreover, the dimension of $\mathfrak{\Re}$ can be arbitrarily large.

Proof. It suffices to imbed $\mathfrak{N}$ in a Lie algebra $\mathfrak{N}_{V}$ of the sort used in Theorem 4.1. We follow the original proof of the Birkhoff imbedding theorem (see [4]). Choose a homogeneous basis for $\mathscr{Q}(\mathfrak{N})$ as in $\S 1$. Let $\mathscr{Q}_{j}=\operatorname{span}\{X: d(X)=j\}$. Given $m>k$, let $X_{m}$ be some nonzero element of degree $m$, let $W_{m}$ be a complementary subspace to span $\left(X_{m}\right)$ in $\mathcal{Q}_{m}$, and let $V=\mathcal{Q}(\Re) /\left[W_{m} \oplus\left(\oplus_{j>m} \mathcal{Q}_{j}\right)\right], V_{j}=$ image of $\mathcal{U}_{j}$ in $V$. $\mathfrak{N}$ acts on $V$ by left multiplication, and $\mathfrak{N}_{j}$ maps $V_{i}$ into $V_{i+j}$. Hence $\mathfrak{N}$ imbeds into $\mathfrak{N}_{V}$ in a way that extends the dilations.

COROLlary 2.3. Any Lie algebra $N$ imbeds in a special Lie algebra.

Proof. The Birkhoff Imbedding Theorem shows that $\mathfrak{N}$ imbeds in a Lie algebra $\mathfrak{N}_{v}, V=\bigoplus_{j=0}^{k} V_{n}$, with $\operatorname{dim} V_{j}=1$ for all $j$.

Notes. (1) Theorem 2.1 also shows that for every $k$, there are $k$-step Lie algebras with square-integrable representations. This confirms an assertion made in [26].

(2) The $\tilde{\mathfrak{R}}$ all have discrete, cocompact subgroups, since the structure constants in the given basis are rational. See [25].

3. Some auxiliary lemmas. This section is devoted to some lemmas concerned with factoring elements of $\delta(N)$. The results will be useful in the next section. The proofs are related to some found in [10], where far more delicate results are proved. In this section, we do not need to assume that $N$ has dilations.

LEMma 3.1. Let $N$ be a nilpotent Lie group, with Lie algebra $\mathfrak{N}$; let $\left\{X_{1}, \ldots, X_{n}\right\}$ be a basis for $\mathfrak{N}$. Given any $k>0$, there exist elements $P_{k}, P_{k}^{\prime} \in \mathcal{U}(\mathfrak{N})$ and functions $f, g \in \mathcal{L}_{1}(N) \cap \mathcal{L}_{2}(N)$ such that:

(1) if $X=X_{1}^{j_{1}} \cdots X_{n}^{j_{n}}$, with $\sum_{i=1}^{n} j_{i} \leqslant k$, then $X * f, f * X, x * g, g * X$ are all in $\mathcal{L}_{1}(N) \cap \mathcal{L}_{2}(N)$

(2) $P_{k} * f=g * P_{k}^{\prime}=\delta_{1}$.

Proof. We shall find $P_{k}$ and $f$; the proof of the existence of $P_{k}^{\prime}$ and $g$ is virtually the same. Since $X * f=X^{t} f$ (where $X^{t}$ is right invariant) and $f * X=X^{t} f$ (where $X^{t}$ is now left invariant), we may deal with differential operators rather than convolutions. Next, the left invariant operators of order $\leqslant k$ can be written as linear combinations (with polynomial coefficients) of the right invariant operators of order $\leqslant k$. If we produce a function $f$ such that $X f$ vanishes rapidly at $\infty$ whenever $X$ is a right invariant operator of order $\leqslant k$, then $f$ will satisfy condition (1). 
The proof is by induction on $n=\operatorname{dim} N$; we begin by reducing the inductive step to the case $n=1$, and then deal with this case. Thus we assume the lemma for $n=1$ and for $n-1$. Since the lemma is independent of the choice of basis, we may assume that $X_{1}, \ldots, X_{n-1}$ span an ideal $\mathfrak{R}_{0}$ of codimension 1 in $N$. Let $N_{0}=\exp \mathfrak{R}_{0}$. A typical element of $N$ can then be written as $x=\left(\exp t X_{n}\right) y=(t, y), t \in R$ and $y \in N_{0}$. Let $x_{t}=(t, 0)$. If $\phi \in e^{\prime}(N)$, then define $\phi_{t}$ on $N_{0}$ by $\phi_{t}(y)=\phi(t, y)$; we have

$$
\begin{aligned}
X_{j} \phi(t, y) & =\left(\left(\operatorname{Ad} x_{t}\right) X_{j}\right)\left(\phi_{t}(y)\right), \quad j<n ; \\
X_{n} \phi(t, y) & =\frac{\partial \phi}{\partial t}(t, y) .
\end{aligned}
$$

Suppose that $f_{0}, P_{0, k}$ satisfy the hypotheses of the lemma on $N_{0}$ and that $f_{1}, P_{1, k}$ satisfy the hypotheses on $\mathbf{R}$. (We assume also that the derivatives of $f_{0}$ and $f_{1}$ of order $\leqslant k$ vanish rapidly at $\infty$.) Define $f$ by

$$
f(t, y)=f_{1}(t) f_{0}\left(x_{t}^{-1} y x_{t}\right) .
$$

Then $X_{j} f(t, y)=f_{1}(t) X_{j} f_{t}(y)$ for $1 \leqslant j \leqslant n$, and $X_{n} f(t, y)=f_{1}(t)\left[X, Y_{t}\right] f(t, y)$, where $\exp Y_{t}=x_{t}^{-1} y x_{t}$. Hence all derivatives of order $\leqslant k$ are in $\mathcal{L}_{1}(N) \cap \mathcal{L}_{2}(N)$, and even vanish rapidly; see $\S 1.3$ of [16]. Moreover, $P_{0, k} P_{1, k} f=\delta$.

For the case $n=1,\left(d^{2} / d t^{2}-1\right)\left(\frac{1}{2} e^{-|t|}\right)=\delta$. Let $P_{k}=\left(d^{2} / d t^{2}-1\right)^{k}$, and let $f$ be the $k$-fold convolution of $\frac{1}{2} e^{-|t|}$ with itself. This completes the proof.

Corollary 3.2. Given $s \geqslant 0$, we can find functions $f, g \in \mathcal{L}_{1}^{s}(N) \cap \mathcal{L}_{2}^{s}(N) \cap$ ${ }^{s} \mathfrak{L}_{1}(N) \cap{ }^{s} \mathfrak{L}_{2}(N)$ and differential operators $P_{s}, P_{s}^{\prime}$ such that $P_{s} g=\delta$ (where $P_{s}$ is left invariant) and $P_{s}^{\prime} f=\delta$ (where $P_{s}^{\prime}$ is right invariant).

Corollary 3.3. Given $s \geqslant 0$, we can find $f, g \in \mathcal{H}^{s}(N)$ and $P_{s}, P_{s}^{\prime} \in U(N)$ such that $P_{s} g=\delta\left(P_{s}\right.$ is left invariant $)$ and $P_{s}^{\prime} f=0$ (where $f$ is right invariant).

LEMMA 3.4. Given $s \geqslant 0$, we can find functions $f, g \in{ }^{s} \varrho_{1}(N) \cap{ }^{s} \varrho_{2}(N) \cap \mathcal{L}_{1}^{s}(N)$ $\cap \mathcal{L}_{2}^{s}(N)$, plus an integer $r$, such that if $\phi \in \mathcal{H}^{r}(N)$, then $\phi=\phi_{1} * f=g * \phi_{2}$, where $\phi_{1}, \phi_{2}$ depend continuously on $\phi$.

Proof. Suppose that $\phi \in \delta(N)$. Then $\phi=\phi * \delta=\phi *\left(P_{k}^{t} * f\right)=\left(\phi * P_{k}^{t}\right) * f=$ $\phi_{1} * f$. (Here, $f$ is as in Corollary 3.2.) The maps $\phi \mapsto \phi_{1}$ and $\phi_{1} \mapsto \phi_{1} * f$ are both continuous in $\mathcal{S}$, and there exists $q$ such that $\phi \mapsto \phi_{1}$ is always continuous from $\mathcal{H}^{r}(N)$ to $\mathcal{K}^{r-q}(N)$. Choose $r$ such that $\phi_{1} \mapsto \phi_{1} * f$ is continuous on $\mathcal{H}^{r-q}(N)$. Then $\phi \mapsto\left(\phi * P_{n}^{t}\right) \mapsto\left(\phi * P_{k}^{t}\right) * f$ is continuous on $\mathcal{H}^{r}(N)$ and is the identity on a dense subset of $\mathcal{K}^{r}(N)$. Hence $\left(\phi * P_{k}^{t}\right) * f=\phi, \forall \phi \in \mathcal{L}_{r}^{2}(N)$, and $\phi \mapsto \phi_{1}=\phi * P_{k}^{t}$ $=P_{k} \phi$ is a continuous map. The proof for $g * \phi_{2}$ is similar, but easier.

LEMMA 3.5. Let $f \in{ }^{s} \mathcal{L}_{1}(N)$, and let $\pi$ be an irreducible representation of $N$. Then: (a) $\pi(f): \mathcal{H}_{\pi} \rightarrow \mathcal{H}_{\pi}^{s}$ is continuous;

(b) there is a constant $c$, independent of $f$ and $\pi$, such that $\|\pi(f)\| \leqslant c_{s}\|f\|_{1}$ (here, $f$ is regarded as an operator from $\mathcal{H}_{\pi}$ to $\mathcal{H}_{\pi}^{s}$ ).

Proof. Let $X \in \mathfrak{N}$ and let $v \in \mathcal{H}_{\pi}$. Then

$$
\|\pi(X) \pi(f) v\|=\|\pi(X * f) v\| \leqslant\|X * f\|_{1}\|v\|_{0} .
$$


Therefore

$$
\|\pi(f) v\|_{s}^{2} \leqslant \sum_{d(X) \leqslant s}\|X * f\|_{1}^{2}\|v\|_{0}^{2},
$$

and $\Sigma_{d(X) \leqslant s}\|X * f\|_{1}^{2}={ }_{s}\|f\|_{1}^{2}$, up to a constant.

COROLlARY 3.6. If $g \in \mathcal{L}_{1}^{s}(N)$ and $\pi$ is an irreducible representation of $N$, then:

(a) $\pi(f): \mathcal{H}_{\pi}^{-s} \rightarrow \mathcal{H}_{\pi}$ is continuous;

(b) there is a constant $c$, independent of $g$ and $\pi$, such that $\|\pi(g)\| \leqslant\|g\|_{1, s}$ (here, $g$ is regarded as an operator from $\mathcal{H}_{\pi}^{-s}$ to $\mathcal{H}_{\pi}$ ).

Proof. Let $f(x)=g(-x)$. Then $\pi(g)=\pi(f)^{t}$ (where $\left.\pi(f): \mathcal{H}_{\pi} \rightarrow \mathcal{H}_{\pi}^{s}\right)$, and $\|g\|_{1, s}={ }_{s}\|f\|_{1}$. Now use Lemma 3.5.

4. Necessary conditions for tempered fundamental solutions. In this section, we prove Theorem 1.2. We begin with an easy result.

LEMMA 4.1. Let $N$ be a nilpotent Lie group, and let $L$ be a left invariant differential operator on $N$. The following conditions on $L$ are equivalent:

(a) If $f \in \mathcal{S}(N)$, then $\exists u \in \mathcal{S}^{\prime}(N)$ with $L u=f$.

(b) L has a tempered fundamental solution.

(c) $\exists s \geqslant 0$ such that $L_{m}$, regarded as an operator from $\mathcal{H}^{-s}(N)$ to $\mathcal{H}^{s}(N)$, has a bounded right inverse.

(d) $\exists s \geqslant 0$ such that $L^{t}$, regarded as an operator from $\mathcal{H}^{-s}(N)$ to $\mathcal{H}^{s}(N)$, has a bounded left inverse.

(e) $\exists s \geqslant 0$ and $C>0$ such that for all $\phi \in \mathcal{S}$,

$$
\|\phi\|_{-s} \leqslant C\left\|L^{t} \phi\right\|_{s} \text {. }
$$

Proof. It is easy to see that (b) $\Rightarrow(a),(c) \Leftrightarrow(d)$, and (d) $\Leftrightarrow(e)$. Furthermore, (c) $\Rightarrow$ (b). For if (c) holds, let $f$ and $P_{s}$ be as in Corollary 3.3. Find $u \in \mathcal{K}^{-s}(N)$ with $L u=f$. Then $L\left(P_{k} * u\right)=P_{k}(L u)=P_{k} f=\delta$, and $P_{k} * u$ is the tempered fundamental solution.

It therefore suffices to prove that (a) $\Rightarrow(e)$. For this, we imitate the proof of Lemma 6.1.2 in [18]. Let $\delta_{0}$ have the same elements as $\delta$, but with a topology given by the seminorms $\left|L^{t} \phi\right|_{s}$, and consider the bilinear form on $\delta \times \mathcal{S}_{0}$ defined by

$$
(f, g)=\int_{\mathbf{R}} f g d x
$$

For fixed $g$, this is obviously continuous in $f$. If $f$ is fixed, choose $u \in \mathcal{S}^{\prime}$ with $L u=f ;$ then

$$
(f, g)=\int_{\mathbf{R}} g \cdot L u d x=\int_{\mathbf{R}} u \cdot L^{t} g d x
$$

is continuous in $g$. Since $\delta$ is Fréchet, this form is continuous. (One reference is [35]; see the corollary to Theorem 34.1.) Therefore $\exists s, C$ :

$$
\left|\int f g d x\right|<C|f|_{s}\left|L^{t} g\right|_{s} .
$$

Fix $g$. Since $|g|_{-s}=\sup _{\left|f_{s}\right|=1}\left|\int f g d x\right|$, (e) follows. 
LEMMA 4.2. Let $N$ be a special nilpotent Lie group, and let $L$ be a homogeneous left invariant differential operator such that $L^{t}: \mathcal{H}^{-s_{0}}(N) \rightarrow \mathscr{H}^{s_{0}}(N)$ has a bounded left inverse. Then $\exists$ s such that for all $\lambda \neq 0, \pi_{\lambda}(L)_{m}: \mathcal{H}_{\lambda}^{-s} \rightarrow \mathcal{H}_{\lambda}^{s}$ has a bounded right inverse.

Proof. The hypothesis is equivalent to the assumption that (4.1) holds for $s=s_{0}$; once (4.1) holds for some $s=s_{0}$, it holds for all $s>s_{0}$.

Write $N=N_{2} \times M_{1} \times Z$ as in $\S 2$, and let $f=\left(f_{2}, f_{1}, f_{0}\right)$ as described there. Restrict $f_{0}$ so that supp $\hat{f_{0}} \subseteq\left[\frac{1}{2}, 2\right]$. Then the map $\beta$ of $\$ 2$ takes $f$ to $\hat{f}=$ $\hat{f_{2}}\left(x_{2}\right) \hat{f_{1}}(l) \hat{f_{0}}(\lambda)$ and gives an equivalence of the right regular representation $R$ with $\hat{R}$, where

$$
\hat{R}(x) \hat{f^{\prime}}\left(x_{2}, l, \lambda\right)=\left(\pi_{\lambda}(x) \hat{f_{1}}\right)\left(x_{2}\right) \hat{f_{1}}(l) \hat{f_{0}}(\lambda) .
$$

In particular, since $L^{t} f=f * L=R\left(L^{t}\right) f$, we get

$$
\hat{R}\left(L^{t}\right)=\lambda^{m / m_{0}}\left(\pi_{1}\left(L^{t}\right) \hat{f_{2}}\right)\left(x_{2}\right) \hat{f_{1}}(l) \hat{f_{0}}(\lambda) .
$$

On the functions $f$ under consideration, $\mathscr{F}$ is continuous as a map into $\delta$, and so there are $s^{\prime}, C^{\prime}$ such that

$$
\left|\hat{R}\left(L^{t}\right) \hat{f}\right|_{s^{\prime}} \geqslant C^{\prime}|\hat{f}|_{-s^{\prime}}
$$

But now (3.2) implies that

$$
\left|\pi_{1}\left(L^{t}\right) f_{2}\right|_{s^{\prime}} \geqslant C^{\prime}\left|f_{2}\right|_{-s^{\prime}}
$$

Finally, since the ordinary Sobolev seminorms on $\mathcal{S}\left(N_{2}\right)$ and the $\mathcal{H}_{1}^{s}$ seminorms are equivalent, we can find $s$ and $C$ with

$$
\left\|\pi_{1}\left(L^{t}\right) \phi\right\|_{s} \geqslant C\|\phi\|_{-s} \text { on } \mathcal{H}_{\lambda}^{\infty} .
$$

This implies that $\pi_{1}\left(L^{t}\right)$ has a bounded left inverse from $\mathcal{K}_{1}^{s}$ to $\mathcal{K}_{1}^{-s}$; by duality, $\pi_{1}(L)_{m}: \mathcal{H}_{1}^{-s} \rightarrow \mathcal{H}_{1}^{s}$ has a bounded right inverse. Since $\pi_{\lambda}(L)$ is a scalar multiple of $\pi_{1}(L)$ when $\lambda>0$, the same result holds for $\lambda>0$. The same argument clearly works for $\lambda<0$.

Lemmas 4.1 and 4.2 evidently imply Theorem 1.2.

5. Sufficient conditions for semiglobal solvability and for tempered fundamental solutions. In this section, we first prove a theorem showing that certain differential operators on nilpotent Lie groups are semiglobally solvable. (For this theorem, the group need not be special.) We then apply the result to special nilpotent Lie groups.

We need a quick summary of Kirillov theory (see [20] or [27] for details). Let $\hat{N}$ be the space of (equivalence classes of) irreducible representations of $N$; then $\hat{N}$ is parametrized by the $\operatorname{Ad}^{*}(N)$-orbits of $\mathfrak{N}^{*}$. There exist a subspace $V$ of $\mathfrak{N}^{*}$ and a Zariski-open subset $\mathfrak{V}$ of $V$ such that "almost all" representations of $N$ are parametrized by $\mathscr{V}$, in the following sense: $\operatorname{Ad}^{*}(N) \mathcal{V}$ is Zariski-open in $\mathfrak{N}^{*}, \mathcal{V}$ is a cross-section for the orbits in $\operatorname{Ad}^{*}(N) \mathscr{V}$, and the representations corresponding to these orbits are sufficient for the Plancherel Theorem. In fact, let $\pi_{\lambda}$ be the irreducible representation corresponding to $\lambda \in \mathcal{V}$. Then there is a rational function $P f$ on $\mathfrak{R}, \operatorname{Ad}^{*}(N)$-invariant and finite on $\mathfrak{V}$, such that for all $\phi \in \mathcal{L}^{1}(N) \cap \mathcal{L}^{2}(N)$,

$$
\|\phi\|_{2,0}^{2}=\int_{\mathscr{V}}|P f(\lambda)| \operatorname{Tr}\left(\pi_{\lambda}(\phi)^{*} \pi_{\lambda}(\phi)\right) d \lambda .
$$


Indeed, $P f$ is polynomial, and can be determined explicitly; see [28] and [6]. We shall refer to the representations $\pi_{\lambda}, \lambda \in \mathcal{T}$, as the representations in general position. The Hilbert space for $\pi_{\lambda}$ is $\mathcal{H}_{\lambda}$, and the Sobolev spaces are $\mathcal{H}_{\lambda}^{s}$. Note that if $N$ is special, then $V \cong R\left(V\right.$ is complementary to $\left.Y^{\perp}\right)$ and $\mathcal{V}=V-\{0\}$.

We shall use some results found in Chapter 4 of [9]. Let $P_{1}, \ldots, P_{k}$ be polynomials on $V$. We can find a polynomial $Q$ on $V$ such that $Q P_{1}, \ldots, Q P_{k}$ extend to $\operatorname{Ad}^{*}(N)$-invariant polynomials on $N^{*}$. Thus there are elements $D_{1}, \ldots, D_{k} \in$ $z(\mathcal{U}(\mathfrak{N}))$ such that

$$
\pi_{\lambda}\left(D_{j}\right)=Q(\lambda) P_{j}(\lambda), \quad \forall \lambda \in \mathscr{T} .
$$

TheOrem 5.1. Let $L \in \mathcal{U}(\mathfrak{N})$. Suppose that there are numbers $s$ and $C$, plus polynomials $P_{1} \neq 0$ and $P_{2}$, such that for all $\pi_{\lambda}$ in general position:

(1) $\pi_{\lambda}\left(L_{m}\right): \mathcal{H} \lambda_{1}^{-s} \rightarrow \mathcal{H} \lambda_{1}^{s}$ has a bounded right inverse, $B_{\lambda}$;

(2) the $B_{\lambda}$ vary measurably with $\lambda$;

(3) $\left|P_{1}(\lambda)\right|\left\|B_{\lambda}\right\| \leqslant C\left|P_{2}(\lambda)\right|$.

Then $L$ is semiglobally solvable.

Note. The $\pi_{\lambda}$ can (almost) all be modeled on the same Hilbert space so that $(\lambda, x) \mapsto \pi_{\lambda}(x)$ is jointly continuous. This explains the meaning of (2). In (3), of course, the norm of $B_{\lambda}$ is as an operator from $\mathcal{H}_{\lambda}^{s}$ to $\mathcal{H}_{\lambda}^{-s}$.

Proof. Let $Q, D_{1}$, and $D_{2}$ be such that $D_{1}, D_{2} \in z(\mathcal{U}(\Re))$ and, for $j=1,2$, $\pi_{\lambda}\left(D_{j}\right)=Q(\lambda) P_{j}(\lambda)$, all $\lambda \in \mathcal{V}$. We shall show that if $\phi \in \delta(N)$, then there is a $c^{\infty}$ function $u$ on $N$ such that $L u=D_{2}^{t} \phi$. Since $D_{2}^{t}$ is semiglobally solvable, the theorem follows easily.

We define $u=u(\phi)$ as a distribution as follows: let $\phi_{0}(-x)=\phi(x)$, and define

$$
(u, \psi)=\int_{\mathscr{V}}|P f(\lambda)| P_{1}(\lambda) Q(\lambda) \operatorname{Tr}\left(\pi_{\lambda}(\psi) B_{\lambda} \pi_{\lambda}\left(\phi_{0}\right)\right) d \lambda, \quad \psi \in \mathcal{S}(N) .
$$

The first task is to see that this makes sense. Write $\psi_{0}=\psi_{1} * f, \phi_{0}=g * \phi_{2}$, where $f \in{ }_{s} \mathcal{L}_{1}(N) \cap \mathcal{L}_{1, s}(N), g \in{ }_{s} \mathcal{L}_{1}(N) \cap \mathcal{L}_{1, s}(N)$, and $\psi_{1}, \phi_{2} \in S(N)$; see Lemma 3.4. Then $\pi_{\lambda}(f)$, regarded as an operator from $\mathcal{K}_{\lambda}^{0}$ to $: \mathfrak{K}_{\lambda}^{*}$. is bounded, with a norm independent of $\lambda$; similarly, $\pi_{\lambda}(g)=\mathcal{H}_{\lambda}^{-s} \rightarrow \mathcal{F}_{\lambda}^{0}$ is bounded independently of $\lambda$. (See Lemma 3.5 and Corollary 3.6.1.) Let $B_{\lambda}^{\prime}=\pi_{\lambda}(g) B_{\lambda} \pi_{\lambda}(f)$. Then $B_{\lambda}^{\prime}=\mathcal{H}_{\lambda} \rightarrow \mathcal{H}_{\lambda}$ is bounded, and

$$
\left\|P_{1}(\lambda)\right\|\left\|B^{\prime}\right\| \leqslant C\left|P_{2}(\lambda)\right|, \quad \forall \lambda \in \mathcal{V} .
$$

(This constant $C$ may be different from the one in the hypothesis.) Hence

$$
\operatorname{Tr}\left(\pi_{\lambda}\left(\psi_{0}\right) B_{\lambda} \pi_{\lambda}(\phi)\right)=\operatorname{Tr}\left(\pi_{\lambda}\left(\psi_{1}\right) B_{\lambda}^{\prime} \pi_{\lambda}\left(\phi_{2}\right)\right),
$$

and this trace exists because $\pi_{\lambda}\left(\psi_{1}\right)$ and $\pi_{\lambda}\left(\phi_{2}\right)$ are both trace class operators. Write \|\|$_{\mathrm{Tr}},\|\|_{\mathrm{H}-\mathrm{S}}$ for the trace class and Hilbert-Schmidt norms respectively. Then

$$
\begin{aligned}
\mid P_{1}(\lambda) Q(\lambda) \| & \operatorname{Tr}\left(\pi_{\lambda}\left(\psi_{1}\right) B_{\lambda}^{\prime} \pi_{\lambda}\left(\phi_{2}\right)\right)|=| P_{1}(\lambda) Q(\lambda)\left|\operatorname{Tr}\left(B_{\lambda}^{\prime} \pi_{\lambda}\left(\phi_{2}\right) \pi_{\lambda}\left(\psi_{\mathrm{i}}\right)\right)\right| \\
& \leqslant\left|P_{1}(\lambda) Q(\lambda)\right|\left\|B_{\lambda}^{\prime}\right\|\left\|\pi_{\lambda}\left(\phi_{2}\right) \pi_{\lambda}\left(\psi_{1}\right)\right\|_{\mathrm{Tr}} \\
& \leqslant C\left|P_{2}(\lambda) Q(\lambda)\right|\left\|\pi_{\lambda}\left(\phi_{2}\right)\right\|_{\mathrm{H}-\mathrm{S}}\left\|\pi_{\lambda}\left(\psi_{1}\right)\right\|_{\mathrm{H}-\mathrm{S}} \\
& \leqslant C\left\|\pi_{\lambda}\left(\phi_{2}\right)\right\|_{\mathrm{H}-\mathrm{S}} \cdot\left\|\pi_{\lambda}\left(D_{2}^{t} \psi_{1}\right)\right\|_{\mathrm{H}-\mathrm{S}} .
\end{aligned}
$$


Hence

$$
\begin{aligned}
|(u, \psi)| & \leqslant C \int_{\mathscr{\Upsilon}}|P f(\lambda)|\left\|\pi_{\lambda}\left(\phi_{2}\right)\right\|_{\mathrm{H}-\mathrm{S}}\left\|\pi_{\lambda}\left(D_{2}^{t} \psi_{1}\right)\right\|_{\mathrm{H}-\mathrm{S}} d \lambda \\
& \leqslant C\left(\int_{\mathscr{\Upsilon}}|P f(\lambda)|\left\|\pi_{\lambda}\left(\phi_{2}\right)\right\|_{\mathrm{H}-\mathrm{S}}^{2} d \lambda\right)^{1 / 2}\left(\int_{\widetilde{T}}|P f(\lambda)|\left\|\pi_{\lambda}\left(D_{2}^{t} \psi_{1}\right)\right\|_{\mathrm{H}-\mathrm{S}}^{2} d \lambda\right)^{1 / 2} \\
& =C\left\|\phi_{2}\right\|_{2,0}\left\|D_{2}^{t} \psi_{1}\right\|_{2,0},
\end{aligned}
$$

so that the integral is finite. Indeed, since $\exists r:\left\|D_{2}^{t} \psi_{1}\right\|_{2,0} \leqslant|\psi|_{r}$, we see that $u \in$ $\mathcal{K}_{2}^{-r}(N)$ for some $r$ (independent of $\phi$ ).

Now let $D \in \mathcal{Q}(\mathfrak{R})$. Then

$$
\begin{aligned}
(U(\phi), D * \psi) & =\int_{\mathscr{V}}|P f(\lambda)| P_{1}(\lambda) Q(\lambda) \operatorname{Tr}\left(\pi_{\lambda}\left(D * \psi_{1}\right) B_{\lambda}^{\prime} \pi_{\lambda}\left(\phi_{2}\right)\right) d \lambda \\
& =\int_{\mathscr{V}}|P f(\lambda)| P_{1}(\lambda) Q(\lambda) \operatorname{Tr}\left(B_{\lambda}^{\prime} \pi_{\lambda}\left(\phi_{2}\right) \pi_{\lambda}(D) \pi_{\lambda}\left(\psi_{1}\right)\right) d \lambda \\
& =\int_{\mathscr{V}}|P f(\lambda)| P_{1}(\lambda) Q(\lambda) \operatorname{Tr}\left(B_{\lambda}^{\prime} \pi_{\lambda}\left(\phi_{2} * D\right) \pi_{\lambda}\left(\psi_{1}\right)\right) d \lambda \\
& =(u(\phi * D), \psi),
\end{aligned}
$$

or $D^{t} * u(\phi)=u(\phi * D)$. Hence $u(\phi)$ has weak derivatives of all orders in $\mathcal{H}_{2}^{-r}(N)$ for some $r$, and so $u \in C^{\infty}(N)$. Finally,

$$
\begin{aligned}
(L(u), \psi) & =\left(u, L^{i} \psi\right)=(u, \psi * L) \\
& =\int_{\mathscr{V}}|P f(\lambda)| P_{1}(\lambda) Q(\lambda) \operatorname{Tr}\left(\pi_{\lambda}(\psi * L) B_{\lambda} \pi_{\lambda}\left(\phi_{0}\right)\right) d \lambda \\
& =\int_{\mathscr{V}}|P f(\lambda)| P_{1}(\lambda) Q(\lambda) \operatorname{Tr}\left(\pi_{\lambda}(\psi) \pi_{\lambda}(L) B_{\lambda} \pi_{\lambda}\left(\phi_{0}\right)\right) d \lambda \\
& =\int_{\mathscr{V}}|P f(\lambda)| P_{1}(\lambda) Q(\lambda) \operatorname{Tr}\left(\pi_{\lambda}(\psi) \pi_{\lambda}\left(\phi_{0}\right)\right) d \lambda \\
& =\int_{\mathscr{V}}|P f(\lambda)| \operatorname{Tr}\left(\pi_{\lambda}(\psi) \pi_{\lambda}\left(D_{1}\right) \pi_{\lambda}\left(\phi_{0}\right)\right) d \lambda \\
& =\int_{\mathscr{V}}|P f(\lambda)| \operatorname{Tr}\left(\pi_{\lambda}(\psi) \pi_{\lambda}\left(D_{1}^{t} * \phi_{0}\right)\right) d \lambda \\
& =\int_{\mathscr{V}}|P f(\lambda)| \operatorname{Tr}\left(\pi_{\lambda}(\psi) \pi_{\lambda}\left(\left(D_{1}^{t} * \phi_{0}\right)\right)\right)^{*} d \lambda \quad\left(\tilde{f}(x)=f\left(\overline{x^{-1}}\right)\right) \\
& =\left\langle\psi,\left(D_{1}^{t} * \phi_{0}\right)^{\sim}\right\rangle \quad(\operatorname{Plancherel}) \\
& =\int_{N} \psi(x)\left(D_{1}^{t} * \phi_{0}\right)\left(x^{-1}\right) d x=\int \psi(x)\left(D_{1}^{t} \phi\right)(x) d x=\left(D_{1}^{t} \phi, \psi\right) .
\end{aligned}
$$

That is, $L u=D_{1}^{t} \phi$, and the proof is finished. Notice that $\pi_{\lambda}(L)$ is being applied only to $C^{\infty}$ vectors in this proof, so that we can use $\pi_{\lambda}(L)$ or $\pi_{\lambda}(L)_{m}$ in the various manipulations.

Note. In fact, $B_{\lambda}$ need exist only for almost all $\lambda \in \mathcal{V}$, provided that conditions (2) and (3) are met. The proof is the same. 
Now we consider the case where $N$ is a special nilpotent Lie group of dimensions $2 d+1$ and $L$ is homogeneous of degree $m_{0}$. Let $a>0$; let $m$ be the homogeneous degree of $Y$. (Recall that $Y$ spans $z(\mathfrak{N})$.) If $\pi_{\lambda}(L)_{m}$ has a bounded right inverse $B_{\lambda}$, then $a^{-m_{0} / m} B_{\lambda}$ is a right inverse for $\pi_{a \lambda}(L)_{m}$. Moreover, as operators from $\mathcal{H}_{a \lambda}^{s}$ to $\mathcal{H}_{a \lambda}^{-s}$ and $\mathcal{H}_{\lambda}^{s}$ to $\mathcal{H}_{\lambda}^{-s}$, respectively,

$$
\left\|B_{a \lambda}\right\| \leqslant\left(a^{-\left(2 s+m_{0}\right) / m}+1\right)\left\|B_{\lambda}\right\| .
$$

Hence if $\pi_{\lambda}(L)_{m}$ has a bounded right inverse $B_{\lambda}: \mathcal{H}_{\lambda}^{s} \rightarrow \mathcal{H}_{\lambda}^{-s}$ for one positive and one negative value of $\lambda$, then Theorem 5.1 applies, and $L$ is locally solvable. This completes the proof of Theorem 1.1.

6. Sufficient conditions for tempered fundamental solutions. In the case of certain homogeneous operators on special nilpotent Lie groups, we can extend Theorem 5.1.

THEOREM 6.1. Suppose that $N$ is special, that $L$ is homogeneous of degree $m_{0}$, and that $\pi_{\lambda}(L)_{m}: \mathcal{H}_{\lambda}^{-s}(N) \rightarrow \mathcal{H}_{\lambda}^{s}(N)$ has a bounded right inverse for every $\lambda \neq 0$. Suppose further that $m_{0}+2 s<m$, where $m$ is the homogeneous degree of $Y$ ( $Y$ is a nonzero central element in $N$ ). Then $L$ has a tempered fundamental solution.

Proof. Let $B_{1}, B_{-1}$ be bounded right inverses for $\pi_{1}(L), \pi_{-1}(L)$ respectively; let $a>0$. As we saw in $\S 5, B_{a}=a^{-m / m_{0}} B_{1}$ and $B_{-a}=a^{-m_{0} / m} B_{-1}$ are right inverses to $\pi_{a}(L), \pi_{-a}(L)$ respectively. Let $\gamma=-\left(m_{0}+2 s\right) / m$; then

$$
\left\|B_{a}\right\| \leqslant\left(a^{\gamma}+1\right)\left\|B_{1}\right\|
$$

(the norms are as operators from $\mathcal{H}_{a}^{s}$ to $\mathcal{K}_{a}^{-s}$ and $\mathcal{H}_{1}^{s}$ to $\mathcal{K}_{1}^{-s}$ respectively), and similarly for $B_{-a}$.

We follow the proof of Theorem 5.1, setting $D_{2}=I$. The main point to be established is that $U(\phi)$ is indeed a distribution. As in Theorem 5.1, we get

$$
\begin{aligned}
&\left|\operatorname{Tr}\left(\pi_{\lambda}\left(\psi_{1}\right) B_{\lambda}^{\prime} \pi_{\lambda}\left(\phi_{2}\right)\right)\right| \leqslant\left\|B_{\lambda}^{\prime}\right\|\left\|\pi_{\lambda}\left(\phi_{2}\right)\right\|_{\mathrm{H}-\mathrm{S}}\left\|\pi_{\lambda}\left(\psi_{1}\right)\right\|_{\mathrm{H}-\mathrm{S}} \\
& \leqslant C\left(|\lambda|^{\gamma}+1\right)\left\|\pi_{\lambda}\left(\phi_{2}\right)\right\|_{\mathrm{H}-\mathrm{s}}\left\|\pi_{\lambda}\left(\psi_{1}\right)\right\|_{\mathrm{H}-\mathrm{s}} .
\end{aligned}
$$

Thus, if $\operatorname{dim} N=2 d+1$, we need to bound

$$
C \int_{\mathbf{R}-\{0\}}|\lambda|^{d+\gamma}\left\|\pi_{\lambda}\left(\phi_{2}\right)\right\|_{\mathrm{H}-\mathrm{s}}\left\|\pi_{\lambda}\left(\psi_{1}\right)\right\|_{\mathrm{H}-\mathrm{s}} d \lambda .
$$

We separate this integral into two pieces, $\int_{|\lambda| \geqslant 1}$ and $\int_{|\lambda| \leqslant 1}$. For the first integral,

$$
\begin{aligned}
& C \int_{|\lambda| \geqslant 1}|\lambda|^{m+\gamma}\left\|\pi_{\lambda}\left(\phi_{2}\right)\right\|_{\mathrm{H}-\mathrm{S}}\left\|\pi_{\lambda}\left(\psi_{1}\right)\right\|_{\mathrm{H}-\mathrm{S}} d \lambda \\
& \quad \leqslant C \int_{|\lambda| \geqslant 1}|\lambda|^{m}\left\|\pi_{\lambda}\left(\phi_{2}\right)\right\|_{\mathrm{H}-\mathrm{S}}\left\|\pi_{\lambda}\left(\psi_{1}\right)\right\|_{\mathrm{H}-\mathrm{S}} d \lambda \\
& \quad \leqslant C \int_{\mathbf{R}-\{0\}}|\lambda|^{m}\left\|\pi_{\lambda}\left(\phi_{2}\right)\right\|_{\mathrm{H}-\mathrm{S}}\left\|\pi_{\lambda}\left(\psi_{1}\right)\right\|_{\mathrm{H}-\mathrm{S}} d \lambda \\
& \quad \leqslant\left\|\phi_{2}\right\|_{2}\left\|\psi_{1}\right\|_{2},
\end{aligned}
$$


as in Theorem 5.1. As for the other piece,

$$
\begin{aligned}
& C \int_{|\lambda| \leqslant 1}|\lambda|^{m+\gamma}\left\|\pi_{\lambda}\left(\phi_{2}\right)\right\|_{\mathrm{H}-\mathrm{S}}\left\|\pi_{\lambda}\left(\psi_{1}\right)\right\|_{\mathrm{H}-\mathrm{S}} d \lambda \\
& \quad \leqslant C\left(\int_{|\lambda|<1}|\lambda|^{m+\gamma}\left\|\pi_{\lambda}\left(\phi_{2}\right)\right\|_{\mathrm{H}-\mathrm{S}}^{2} d \lambda \cdot \int_{|\lambda|<1}|\lambda|^{m+\gamma}\left\|\pi_{\lambda}\left(\psi_{1}\right)\right\|_{\mathrm{H}-\mathrm{S}}^{2} d \lambda\right)^{1 / 2} .
\end{aligned}
$$

Now

$$
\begin{aligned}
|\lambda|^{m}\left\|\pi_{\lambda}\left(\phi_{2}\right)\right\|_{\mathrm{H}-\mathrm{S}}^{2} & =|\lambda|^{m} \operatorname{Tr}\left(\pi_{\lambda}\left(\tilde{\phi_{2}} * \phi_{2}\right)\right) \quad\left(\tilde{\phi_{2}}(x)=\overline{\phi_{2}(-x)}\right) \\
& =\int_{l(Y)=\lambda}\left(\tilde{\phi_{2}} * \phi_{2}\right)^{\hat{}}(l) d l .
\end{aligned}
$$

Let $\Phi=\phi_{2}^{\tilde{2}} * \phi_{2}$, and regard $\Phi$ as a function on $\mathfrak{N}$ (via exp); then, by Poisson summation,

$$
\left.\int_{l(Y)=\lambda} \Phi^{\wedge}(l) d l=\int_{\mathbf{R}} \Phi(t Y) e^{2 \pi i \lambda t} d t \leqslant \int . \quad \mathbf{v}\right) \mid d t=\alpha(\Phi), \quad \text { say, }
$$

and

$$
\int_{|\lambda| \leqslant 1} \lambda^{d r \gamma}\left\|\pi_{\lambda}\left(\phi_{2}\right)\right\|_{\mathrm{H}-\mathrm{S}}^{2} d \lambda \leqslant \alpha(\Phi) \int_{|\lambda| \leqslant 1}|\lambda|^{\gamma} d \lambda=\frac{2}{1+\gamma} \alpha(\Phi)
$$

Since $\phi_{2} \mapsto \sqrt{\alpha(\Phi)}$ is continuous on $\delta$, we get

$$
\left(\int_{|\lambda| \leqslant 1} \lambda^{d+\gamma}\left\|\pi_{\lambda}\left(\phi_{2}\right)\right\|_{\mathrm{H}-\mathrm{S}}^{2} d \lambda\right)^{1 / 2} \leqslant\|\| \phi \|_{2, r}, \quad \text { some } r \geqslant 0
$$

Similar remarks apply to $\psi_{1}$. Therefore $\phi \mapsto u(\phi)$ is continuous from $\mathcal{H}_{2}^{r}(N)$ to $\mathcal{H}_{2}^{-r}(N)$. Of course, $L u(\phi)=\phi$, as in the proof of Theorem 5.1.

From Corollary 4.3, we can find $f \in \mathcal{H}_{2}^{r}(N)$ and $P^{\prime} \in U(N)$ such that $P_{r}^{\prime} * f=\delta$. Let $\xi=P_{r}^{\prime} * u(f)$. Then, since $L$ is left invariant,

$$
L(\xi)=P_{r}^{\prime} * L(u(f))=P_{r}^{\prime} * f=\delta
$$

and $\xi \in \mathcal{S}^{\prime}$. This proves the theorem.

Theorem 6.1 is obviously much too weak to fill the gap between Theorem 1.1 and the full converse of Theorem 1.2. The following theorem gives a full converse, but in a special case.

Theorem 6.2. Let $N$ be the $(2 n+1)$-dimensional Heisenberg group, and let $L$ be a left invariant operator on $N$ satisfying the hypotheses of Theorem 5.1. Then L has a tempered fundamental solution.

Proof. The Lie algebra $\mathfrak{N}$ of $N$ has a basis $X_{1}, \ldots, X_{n}, Y_{1}, \ldots, Y_{n}, Z$, with $\left[X_{i}, Y_{j}\right]=\delta_{i j},\left[X_{i}, X_{j}\right]=\left[Y_{i}, Y_{j}\right]=0, Z$ central. Moreover, the center of $\mathscr{U}(\mathfrak{R})$ is generated by $Z$. Coordinatize $N$ by using the exponential map from $\mathfrak{R}$; let

$$
(x, y, z)=\left(x_{1}, \ldots, x_{n}, y_{1}, \ldots, y_{n}, z\right)=\exp \left(z Z+\sum_{j=1}^{n}\left(x_{j} X_{j}+y_{j} Y_{j}\right)\right) \text {. }
$$


Then a simple calculation shows that

$$
X_{j}=\frac{\partial}{\partial x_{j}}-\frac{1}{2} y_{j} \frac{\partial}{\partial z}, \quad Y_{j}=\frac{\partial}{\partial y_{j}}+\frac{1}{2} x_{j} \frac{\partial}{\partial z}, \quad Z_{j}=\frac{\partial}{\partial z} .
$$

Next, consider the polynomial $P_{1}(\lambda)$ of Theorem 5.1 corresponding to $L$. (In this case, there is no difficulty in showing that we may take $Q \equiv 1$.) We may assume that it is monic, so that $P_{1}(\lambda)=\Pi_{j=1}^{r}\left(\lambda-\lambda_{j}\right)$. If $\lambda_{j} \notin \mathbf{R}$, then $\left|\lambda-\lambda_{j}\right|$ is bounded below on $\mathbf{R}$, and condition (3) of Theorem 5.1 holds if $P_{1}(\lambda)$ is replaced by $P_{1}(\lambda)\left(\lambda-\lambda_{j}\right)^{-1}$. Thus we may assume that $P_{1}(\lambda)$ has all real roots.

Theorem 5.1, plus the argument at the end of Theorem 6.1, shows that we can find a tempered distribution $u$ such that $L u=P_{1}(Z) \delta$. Since $P_{1}(Z)$ is a constant coefficient operator, Theorem 4.3 of [15] implies that there is a tempered distribution $\xi_{0}$ such that $P_{1}(Z) \xi_{0}=u$. Let $L \xi_{0}=\alpha_{0}$. Then

$$
P_{1}(Z) \alpha_{0}=P_{1}(Z) L \xi_{0}=L P_{1}(Z) \xi_{0}=L u=P_{1}(Z) \delta,
$$

or

$$
P_{1}(Z)\left(\alpha_{0}-\delta\right)=0 .
$$

Set $\alpha=\delta-\alpha_{0}$. Obviously we are done if we can find a tempered distribution $\xi$ such that $L(\xi)=\alpha$. We first show that (6.1) implies that

$$
\exists m \geqslant 0: Z^{m} \alpha=0 .
$$

Because all roots of $P(Z)$ are real, it suffices to prove that if $\lambda_{1} \in \mathbf{R}, \lambda_{1} \neq 0$, and $\left(Z_{1}-\lambda_{1}\right) \alpha=0$, then $\alpha=0$. (Recall that $\alpha$ is a tempered distribution.) Let $\alpha^{\prime}=$ $e^{-\lambda_{1} z} \alpha$; then $Z \alpha^{\prime}=0$. Example 4.5.1 of [34] states that $\alpha^{\prime}=\beta^{\prime}(x, y) \otimes 1$, and hence that $\alpha=\beta^{\prime}(x, y) \otimes e^{-\lambda_{1} z}$. Since $\alpha$ is tempered and $\lambda_{1} \neq 0$, we must have $\alpha=0$.

Write $L=L_{0} Z^{m}$, where $L_{0}$ is not divisible by $Z$ (i.e., $L_{0}$ is not trivial on every function constant under translation by all elements $\exp (z Z))$. If we can solve

$$
L_{0} \eta=\beta, \quad \eta \in \mathcal{S}^{\prime},
$$

whenever $\beta \in \mathcal{S}^{\prime}$ satisfies $Z^{k+m} \beta=0$, then we are done; choose $\beta$ so that $Z^{k} \beta=\alpha$, and we have $L \eta=\alpha$.

For simplicity of notation, replace $(k+m)$ by $m$. Example 4.5.1 of [34], plus a simple induction, shows that we can write

$$
\beta=\sum_{j=0}^{m} \beta_{j}(x, y) \otimes z^{j},
$$

where the $\beta_{j}$ are tempered distributions on $\mathbf{R}^{2 n} \cong$ the $(x, y)$-hyperplane. Let $X_{j}^{\prime}=$ $\partial / \partial x_{j}, Y^{\prime}=\partial / \partial y_{j}$, and define $L_{0}^{\prime}$ as follows: if $L_{0}=P(X, Y, Z)$, write $L_{0}^{\prime}=$ $P\left(X^{\prime}, Y^{\prime}, 0\right)$. By the definition of $L_{0}, L_{0}^{\prime} \neq 0$; it is easy to check that $L_{0}=L_{0}^{\prime}+Z L_{1}$, where $L_{1}$ is a differential operator whose coefficients are polynomials in $x$ and $y$. $\left(L_{1}\right.$ has no particular invariance properties.) Since $L_{0}^{\prime}$ is a constant coefficient operator, we can find a distribution $\eta_{m} \in \mathcal{S}\left(\mathbf{R}^{n}\right)$ such that $L_{0}^{\prime}\left(\eta_{m}\right)=\beta_{m}$. Then

$$
L_{0}\left(\eta_{m} \otimes z^{m}\right)=\sum_{j=0}^{m-1} \beta_{j}^{\prime}(x, y) \otimes z^{j},
$$


where $\beta_{m}^{\prime}=\beta_{m}$. It follows (by induction on $m$ ) that we can solve (6.1), and the proof is complete.

7. Examples and open questions. We begin with a simple example showing that Theorem 1.1 really does apply to some operators for which the corresponding statement with $s=0$ fails. The 3-dimensional Heisenberg group $N_{1}$ has a Lie algebra spanned by $X, Y$, and $Z$, with $[X, Y]=Z$. The representations $\pi_{\lambda}$ are realized on $e^{2}(\mathbf{R})$, with

$$
\pi_{\lambda}(X)=|\lambda|^{1 / 2}(d / d t), \quad \pi_{\lambda}(Y)=|\lambda|^{1 / 2}(\operatorname{sgn} \lambda) \cdot 2 \pi i t I, \quad \pi_{\lambda}(Z)=2 \pi i \lambda I .
$$

Now let $L=Y$. Then $\pi_{\lambda}(Y)$ is not invertible from $\varrho^{2}(\mathbf{R})$ to $\varrho^{2}(\mathbf{R})$. If $s$ is sufficiently large, however, every element of $\mathcal{H}_{\lambda}^{s}$ is a bounded continuously differentiable function, and the principal value distribution $Q$, with

$$
(Q, \phi)=\lim _{\varepsilon \rightarrow 0} \int_{|x|>\varepsilon} \frac{\phi(x)}{x} d x,
$$

is in $\mathcal{H}_{\lambda}^{-s}$. The map $f \mapsto Q f$ is (up to a scalar) the desired inverse for $\pi_{\lambda}(Y)$.

The next example, also on $N_{1}$, is more interesting.

The operator $L=\left(Y^{2}-2 \pi i Z\right) X$ on $N_{1}$ has a tempered fundamental solution. For one can solve $X \xi=\delta$ for a tempered distribution $\xi$ because $X$ is (in appropriate coordinates) a constant coefficient operator. Similarly, because $Y$ and $Z$ commute, $Y^{2}-2 \pi i Z$ is a constant coefficient operator (in appropriate coordinates); Theorem 4.3 of [19] implies that $\left(Y^{2}-2 \pi i Z\right) \eta=\xi$ is solvable in $\mathcal{S}^{\prime}$. Then $\eta$ is a tempered fundamental solution.

For $\lambda>0$,

$$
\pi_{\lambda}\left(L^{t}\right)=4 \pi^{2} \lambda^{3 / 2} \frac{d}{d t}\left(t^{2}+1\right) \circ I ; \quad \pi_{\lambda}(L)=4 \pi^{2} \lambda^{3 / 2}\left(t^{2}+1\right) \frac{d}{d t} .
$$

Thus if $\phi \in \delta\left(N_{1}\right)$ and $f(t)=\left(1+t^{2}\right)^{-1}$,

$$
\left(\pi_{\lambda}(L) \phi, f\right)=0 .
$$

That is, $\pi_{\lambda}\left(L^{t}\right)_{m} f=0$. So $\pi_{\lambda}\left(L^{t}\right)_{m}$ has no left inverse.

The following problems, among others, are still left open:

(1) Does every globally solvable left invariant operator have a fundamental solution?

(2) Does every globally solvable operator have a tempered fundamental solution?

Finally, it would be useful to be able to extend Theorem 1.1 so that it applies to more general operators and groups. Of course, one can apply Theorem 1.1 to arbitrary homogeneous operators by imbedding them in special nilpotent Lie groups, but that procedure is unwieldy and artificial. 


\section{BIBLIOGRAPHY}

1. M. Atiyah, Resolution of singularities and division of distributions, Comm. Pure Appl. Math 23 (1970), 145-150.

2. I. N. Bernstein, The analytic continuation of generalized functions with respect to a parameter, Functional Anal. Appl. 6 (1973), 273-285.

3. I. N. Bernstein and S. Gelfand, The meromorphic function $P^{\lambda}$, Functional Anal. Appl. 3 (1969), $84-86$.

4. G. Birkhoff, Representability of Lie algebras and Lie groups by matrices, Ann. of Math. (2) 38 (1937), 526-532.

5. W. Chang, Global solvability of invariant differential operators, Ph.D. Thesis, M.I.T., 1978.

6. L. Corwin, A representation-theoretic criterion for local solvability of left-invariant differential operators on nilpotent Lie groups, Trans. Amer. Math. Soc. 264 (1981), 113-120.

7. L. Corwin, F. P. Greenleaf and R. Penney, A general character formula for the distribution kernels of primary projections in $L^{2}$ of a nilmanifold, Math. Ann. 225 (1977), 21-37.

8. L. Corwin and L. P. Rothschild, Necessary conditions for local solvability of homogeneous left invariant differential operators on nilpotent Lie groups, Acta Math. 147 (1981), 265-288.

9. J. Dixmier, Algèbres enveloppantes, Gauthier-Villars, Paris, 1974.

10. J. Dixmier and P. Malliavin, Factorisations de fonctions et de vecteurs indefiniment différentiables, Bull. Sci. Math. 102 (1978), 305-330.

11. M. Duflo, Opérateurs différentiels bi-invariants sur un groupe de Lie, Ann. Sci. École Norm. Sup. 10 (1977), 265-288.

12. M. Duflo and D. Wigner, Convexité pour les opérateurs différentiels invariants sur les groupes de Lie, Math. Z. 167 (1979), 61-80.

13. G. Folland, A fundamental solution for a subelliptic operator, Bull. Amer. Math. Soc. 79 (1973), 373-376.

14. . Subelliptic estimates and function spaces on nilpotent Lie groups, Ark. Mat. 13 (1975), 161-208.

15. B. Gaveau, Solutions fondamentales, représentations, et estimées sous-elliptiques pour les groupes nilpotents d'ordre 2, C. R. Acad. Sci. Paris Sér. A 282 (1976), 563-566.

16. R. Goodman, Nilpotent Lie groups: Structure and applications to analysis, Lecture Notes in Math., vol. 562, Springer-Verlag, Berlin, 1976.

17. P. Greiner, J. J. Kohn and E. Stein, Necessary and sufficient conditions for solvability of the Lewy equation, Proc. Nat. Acad. Sci. U.S.A. 72 (1975), 3287-3289.

18. L. Hörmander, Linear partial differential operators, Grundlehren Math. Wiss., vol. 116, SpringerVerlag, Berlin, 1963

19. On the division of distributions by polynomials, Ark. Mat. 3 (1958), 555-568.

20. A. A. Kirillov, Unitary representations of nilpotent Lie groups, Russian Math. Surveys 17 (1962), 53-104.

21. P. Levy-Bruhl, Application de la formule de Plancherel à la resolubilité d'opérateurs invariants a gauche sur des groupes nilpotents d'ordre deux, Bull. Sci. Math. 104 (1980), 369-391.

22. H. Lewy, An example of a smooth linear partial differential equation without solution, Ann. of Math. (2) 66 (1957), 155-158.

23. G. Lion, Hypoellipticité et resolubilité d'opérateurs différentials sur des groupes nilpotents de rang 2 , C. R. Acad. Sci. Paris Ser. A 290 (1980), 271-274.

24. S. Lojasiewicz, Sur le probleme de division, Studia Math. 18 (1959), 87-136.

25. A. I. Malcev, On a class of homogeneous spaces, Amer. Math. Soc. Transl. (1) 39 (1951).

26. C. C. Moore and J. Wolf, Square integrable representations of nilpotent groups, Trans. Amer. Math. Soc. 185 (1973), 445-462.

27. L. Pukanzky, Leçons sur les représentations des groupes, Dunod, Paris, 1967.

28. , On the characters and the Plancherel formula of nilpotent groups, J. Funct. Anal. 1 (1967), 255-280.

29. M. Raïs, Solutions élémentaires des opérateurs différentiels bi-invariants sur un groupe de Lie nilpotent, C. R. Acad. Sci. Paris Sér. A 273 (1971), 595-598.

30. C. Rockland, Hypoellipticity on the Heisenberg group-representation-theoretic criteria, Trans. Amer. Math. Soc. 240 (1978), 1-52. 
31. L. P. Rothschild, Local solvability of left invariant differential operators on the Heisenberg group, Proc. Amer. Math. Soc. 74 (1979), 383-388.

32. L_ Local solvability of second order differential operators on nilpotent Lie groups, Ark. Mat. 19 (1981), 145-175.

33. L. P. Rothschild and D. Tartakoff, Inversion of analytic matrices and local solvability of some invariant differential operators on nilpotent Lie groups, Comm. Partial Differential Equations 6 (1981), 625-650.

34. L. Schwartz, Théorie des distributions, Hermann, Paris, 1966.

35. J. F. Treves, Topological vector spaces, distributions and kernels, Academic Press, New York, 1967.

36. L. Corwin and F. P. Greenleaf, Character formulas and spectra of compact nilmanifolds, J. Funct. Anal. 21 (1976), 123-154.

Department of Mathematics, Rutgers University, New Brunswick, New JerSey 08903 